\title{
The Mechanism Analysis of Underactuated Robotic Finger for Optimum Grasping Using Gradient Descent Method
}

\author{
Sadeq H. Bakhy \\ Enass H. Flaieh \\ Mortada A. Jabbar \\ Mechanical Eng. Dep., University of Technology, Baghdad, IRAQ \\ sadeqbakhy@yahoo.com \\ enass hassen@yahoo.com \\ mortada.mechano@yahoo.com
}

\author{
Received: 12- Oct.-2017Ｒevised: 19-Nov.-2017Ａccepted: 18- Dec.-2017 \\ http://doi.org/10.29194/NJES21010118
}

\begin{abstract}
This study was devoted in investigating the optimum geometric parameters for underactuated linkage three phalanges robotic finger. New kinematic and kinetic equations of grasping were derived in this research taking into account the angle for the ternary solid links of the four-bar linkages. To obtain the target of optimization, a gradient descent method was used which consists of three stages to find the optimal geometric parameters with high accuracy. Five criteria were selected to find the optimal solution by using multi objectives function algorithm, these are percentage of the grasping stability, the grasp forces, squeezing force, Mimic function for grasping task, and transmission angle for grasping operation. Gradient descent method starts by detecting the optimal geometric parameters for each criterion and choosing the best geometric parameters from the five criteria functions. At the optimum solution, the underactuated robotic finger prototype was built from hard Polylactic acid (PLA) plastic using rapid prototyping and was tested performance by grasping objects. Finally, the results have been shown that the robotic finger adapts to the wanted configurations.
\end{abstract}

Keywords: Underactuated, Robotic finger, Gradient descent method, Grasping, four-bar linkages.

\section{Introduction}

Robotic fingers are the main parts of robot hand which are classified into two main kinds: dexterous and underactuated fingers. In spite of the dexterous fingers are graceful but they are very expensive. Underactuated fingers can grasp objects self-adaptively so it makes them low cost and easy to control, inverting the dexterous robotic fingers, the underactuated function makes fingers feel hard to grasp graceful things [1]. Gosselin and Liliberte [2] developed a mechanism consisting of three fingers, each finger has three phalanges. The mechanism contained the orientation of the distal phalanx relatively to the palm in order to make contact between the palm and the object. Each finger of the hand was driven by two actuators, one of these actuator will drive the phalanges and the other to change the orientation of the finger. The transmission from the actuators to the finger was by a ball screw to get large forces to allow the modification of the transmission ratio.

Thierry Lalibert'e et al. [3] developed selfadaptive and reconfigurable hands, which are versatile and easy to control with using linkage mechanism, each hand has a three fingers and each finger has three phalanges. The selfadaptability of the hands can be obtained by using the underactuation. The hand has 12 degrees of freedom and 6 motors, while the second hand has 10 degrees of freedom and 2 motors. Rodrigues et al. [4] introduced the LARM hand prototype that has three degrees of freedom, each finger was consisted of two four-bar linkage mechanisms. The first phalanx was the input bar for the first four-bar linkage mechanism and it's also the frame for the second four bar linkage mechanism. The input bar of the second four-bar linkage mechanism is the second phalanx and it is also the coupler of the first four-bar linkage mechanism. The coupler of the second four-linkage mechanism is the third phalanx.

S. Yao et al. [5] presented two schemes that have been modified in the design. The two mechanisms have three degrees of freedom system and one actuator, the finger has phalanxes that composed of four-bar mechanisms that have linear springs in the joints and also with torsional spring.

Deyang Zhao [6] introduced a self-adaptive finger and two degrees of freedom coupled. Mechanism of the rigid coupled linkage and the self-adaptive for underactuated linkages were combined. The motions can coordinate by the two mechanisms, where the mechanism gets one motion to the other when the finger will touch the object. Decoupling elements including a changing bar and a decoupling spring were integrated in the architecture to decouple the coupled mechanism and enable the underactuated mechanism. The decoupling spring connected the changing bar and the base of the finger.

Jui Hsu et al. [7] presented design of a robotic gripper, which aims to realize the locked and robust grasps. They proposed a solution to design a smart self-locking underactuated mechanism 
mounted in parallel to the actuators to be triggered automatically when the desired grasp is achieved. This design influences the adjustable power distribution between the brake through a differential gear and the gripper.

Jean-Michel Boucher and Lionel Birglen [8] introduced underactuated fingers' grasps using multiple drive actuation. In this study, the implementation augmentation of underactuated fingers through supplemental actuators is presented and discussed. The self-adaptive, fingers typically only depend on a single actuator for a given number of degrees of freedom, mostly equal to the number of phalanges. The research proposed to use more than one actuator to drive underactuated fingers to get better the typical metrics used to measure the grasp performances like stiffness and stability. Typical performance indices are posteriorly presented and optimizations are completed to compare the best designs achievable with respect to stiffness and grasp stability, depending on the number of actuators.

In this paper, new equations of kinetic and kinematic analysis for grasping robotic finger were derived taking into account the angle of the ternary solid links in the four-bar linkages. Also, an underactuation robotic finger has been designed based on multi objectives by using gradient descent method, that are percentage of the grasping stability, the grasp forces, squeezing force, Mimic function for grasping task, and transmission angle for grasping task. In addition, construction of a robotic finger is performed experimentally according to optimal dimensions of fingers and tests performance by grasping objects.

\section{Mechanism of Robotic Finger}

In this research the robotic finger is designed to be able to perform the grasping operation with addition the angle of the ternary solid links between the two four-bar linkages, as shown in figure (1). Mechanism of robotic finger consists of three phalanges which are distal, middle and proximal phalanges. The movement of the robotic finger starts from the slider mechanism by giving the input torque to the system. The solid link of the first four bar linkage which is the input link will accelerate that leads to move the second link (coupler link) to transmit the movement to the output link to accelerate the first phalanx. The output of the first four bar mechanism is the input to the second four bar mechanism that will move the coupler link of the mechanism to transmit the movement to the output link which lead to move the second phalanx. The output of the second four bar mechanism is the input of the third solid link that will be moved the third phalanx.

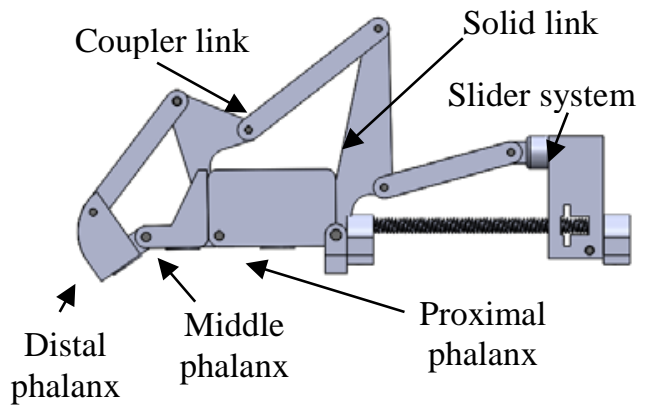

Figure (1): Robotic finger mechanism

\section{Static Kinetic (Kino-static) Analysis of Robotic Finger \\ 3.1 Grasping Force}

Kino-static model is made on the three phalanx finger in order to obtain the grasping forces. The model is shown in figure (2). According to the principle of virtual work the grasping force can be written as [9]:

$$
F^{T}=t_{a}^{T} J_{w}^{-1} J_{v}^{-1}
$$

Where $t^{T}$ the input torque vector exerted by the actuator and the springs mounted between the phalanges. $J_{v}$ is the lower triangular matrix describe jacobian matrix for three phalanx robotic finger considering the friction between the contact points and the object in to the account that can be written as [10]:

$$
\mathrm{J}_{v}=\left[\begin{array}{ccc}
h_{1} & 0 & 0 \\
X & h_{2} & 0 \\
Z & Y & h_{3}
\end{array}\right]
$$

Coefficient of friction $(\mu)$ depends on the material of the object-finger surface pair, numerical values are (1-4) for solid-rubber, conservative value of 2 has been chosen.

In this study, $J_{w}$ is transmission matrix which characterizes the underactuation between the fingers using four-bar linkage mechanism taking account the angle of ternary solid links (i.e. $\gamma_{2}$ ). It can be expressed as:

$$
J_{w}=\left[\begin{array}{ccc}
1 & A & -A B \\
0 & 1 & 0 \\
0 & 0 & 1
\end{array}\right]
$$

Where:

$$
\begin{aligned}
& A=\frac{l_{1} c_{1} \sin \left(\theta_{2}-\Psi_{2}-\gamma_{2}\right)}{l_{1} a_{1} \sin \left(\Psi_{1}\right)+c_{1} a_{1} \sin \left(\Psi_{1}-\Psi_{2}+\theta_{2}-\gamma_{2}\right)}- \\
& \frac{a_{1} c_{1} \sin \left(\Psi_{1}-\Psi_{2}+\theta_{2}-\gamma_{2}\right)}{l_{1} a_{1} \sin \left(\Psi_{1}\right)+c_{1} a_{1} \sin \left(\Psi_{1}-\Psi_{2}+\theta_{2}-\gamma_{2}\right)} \\
& B=\frac{l_{2} c_{2} \sin \left(\theta_{3}-\Psi_{3}\right)}{l_{2} a_{2} \sin \left(\Psi_{2}\right)+a_{2} c_{2} \sin \left(\Psi_{2}-\Psi_{3}+\theta_{3}\right)}- \\
& \frac{a_{2} c_{2} \sin \left(\Psi_{2}-\Psi_{3}+\theta_{3}\right)}{l_{2} a_{2} \sin \left(\Psi_{2}\right)+a_{2} c_{2} \sin \left(\Psi_{2}-\Psi_{3}+\theta_{3}\right)}
\end{aligned}
$$

When the torques exerted by the springs is ignored, the grasping force can be expressed as: 


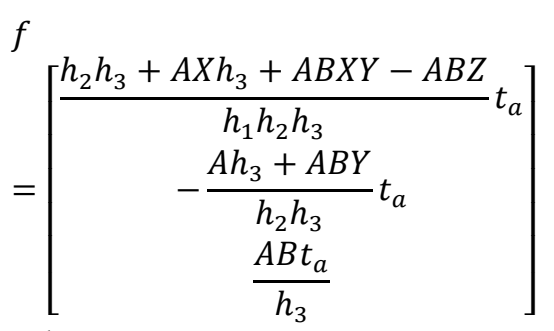

Where:

$X=l_{1}\left(\cos \theta_{2}+\mu \sin \theta_{2}\right)+h_{2} \quad(7)$

$Y=l_{2}\left(\cos \theta_{3}+\mu \sin \theta_{3}\right)+h_{3}$

$Z=l_{1} \cos \left(\theta_{2}+\theta_{3}\right)+l_{1}\left(\cos \theta_{3}\right.$

$$
\left.+\mu \sin \theta_{3}\right)+h_{3}(9)
$$

Noticing equation (4), contain the terms of angle $\left(\gamma_{2}\right)$ which is different from the previous researches (i.e. Ref. [9]). If the angle $\left(\gamma_{2}\right)$ is equal to zero, matrix in equation (3) will be the same as the transmission matrix for the four bar linkage without $\left(\gamma_{2}\right)$. This leads that the new derived form of transmission matrix is more general from the previous one.

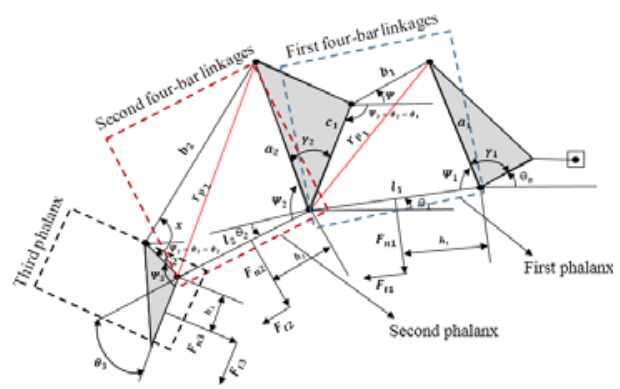

Figure (2): Kino-static model of three-phalanx for robotic finger

\section{Static Kinematic Analysis of Robotic Finger}

\subsection{Kinematics analysis for grasping task}

The phalanges movement are constrained by the object. Two stages are considered the first stage when only the proximal phalanx touches the object, while the second stage when both proximal and the distal phalanges touch the object. At the first stage, the link $l_{1}$ represent the base while angle $\left(\theta_{12}\right)$ is the input angle. As shown in figure (3).

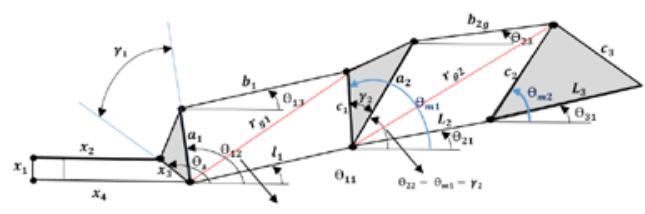

Figure (3): Kinematic model of three-phalanx for robotic finger
It's necessary to find angle $\left(\theta_{s}\right)$ before angle $\left(\theta_{12}\right)$, which represent the output angle of the slider mechanism that can be written as [11]:

$$
\begin{gathered}
\theta_{s}=2 \tan ^{-1}\left[\frac{-B_{s} \pm \sqrt{B_{s}^{2}-C_{s}^{2}+A_{s}^{2}}}{C_{s}-A_{s}}\right] \\
A_{s}=2 x_{3} x_{4} \cos \theta x_{4}-2 x_{1} x_{3} \cos \theta x_{1} \\
B_{s}=2 x_{3} x_{4} \sin \theta_{x 4}-2 x_{1} x_{3} \sin \theta x_{1} \\
C_{s}=x_{1}^{2}+x_{3}^{2}+x_{4}^{2}-x_{2}^{2} \\
-2 x_{1} x_{4}\left(\sin \theta_{x 1} \sin \theta_{x 4}\right. \\
\left.+\cos \theta_{x 1} \cos \theta_{x 4}\right)
\end{gathered}
$$

In this study, $\left(\theta_{12}=\theta_{s}-\gamma_{1}\right)$ is the input angle of the first four bar linkages, as shown in figure (3). $\theta_{m 1}$ represent the output angle of the first four bar linkages, which can be calculated as:

$$
\begin{gathered}
\theta_{m 1}=2 \tan ^{-1}\left[\frac{-B_{1} \pm \sqrt{B_{1}^{2}-C_{1}^{2}+A_{1}^{2}}}{C_{1}-A_{1}}\right] \\
A_{1}=2 l_{1} c_{1} \cos \left(\theta_{11}\right)-2 a_{1} c_{1} \cos \left(\theta_{12}\right) \\
B_{1}=2 l_{1} c_{1} \sin \left(\theta_{11}\right)-2 a_{1} c_{1} \sin \left(\theta_{12}\right) \\
C_{1}=a_{1}^{2}+c_{1}^{2}+l_{1}^{2}-b_{1}^{2} \\
\quad-2 a_{1} l_{1}\left[\cos \left(\theta_{11}\right) \cos \left(\theta_{12}\right)\right. \\
\left.\quad+\sin \left(\theta_{11}\right) \sin \left(\theta_{12}\right)\right]
\end{gathered}
$$

In the second stage $\left(\theta_{22}=\theta_{m 1}-\gamma_{1}\right)$ is the input angle of the second four bar linkages. $\theta_{m 2}$ represent the output angle of the second four bar linkages, which can be calculated as:

$$
\begin{gathered}
\theta_{m 2}=2 \tan ^{-1}\left[\frac{-B_{2} \pm \sqrt{B_{2}^{2}-C_{2}^{2}+A_{2}^{2}}}{C_{2}-A_{2}}\right] \\
A_{2}=2 l_{2} c_{2} \cos \left(\theta_{21}\right) \\
B_{2}=2 l_{2} c_{2} \sin \left(\theta_{21}\right) a_{2} c_{2} \cos \left(\theta_{m 1}-\gamma_{2}\right) \\
C_{2}=
\end{gathered}
$$

$a_{2}^{2}+c_{2}^{2}+l_{2}^{2}-b_{2 g}^{2}-2 a_{2} l_{2}\left[\cos \left(\theta_{21}\right) \cos \left(\theta_{m 1}-\right.\right.$ $\left.\left.\gamma_{2}\right)+\sin \left(\theta_{21}\right) \sin \left(\theta_{m 1}-\gamma_{2}\right)\right]$

From figure (3), the angle $\left(\theta_{21}\right)$ before middle phalanx touches the object can be calculated as:

$$
\begin{aligned}
\theta_{21}= & \theta_{m 1}-\left[\pi-\tan ^{-1}\left(\frac{a_{1}}{l_{1}}\right)-\right. \\
& \left.\cos ^{-1}\left[\frac{b_{1}^{2}-c_{1}^{2}-\left(a_{1}^{2}+l_{1}^{2}\right)}{-2 c_{1} \sqrt{a_{1}^{2}+l_{1}^{2}}}\right]\right]
\end{aligned}
$$

The value of the angle $\theta_{31}$ after the proximal and distal phalanges touch the abject can be calculated:

$$
\theta_{31}=\theta_{m 2}-\left[\cos ^{-1}\left[\frac{c_{3}^{2}-c_{2}^{2}-l_{3}^{2}}{-2 c_{2} l_{3}}\right]\right]
$$

Equations (10-17) were used to find the output angle of the first four bar linkage, as noticed they are the same equations for the four-bar linkage as reference [12], but equations in the present study 
included the angle of the ternary solid link $\left(\gamma_{1}\right)$, as shown in figure (3). Also the angle $\left(\gamma_{2}\right)$ were noticed in equations (18-21) used to find the output angle of the second four bar linkage, as stated before the new forms of the equation is more general than the previous one.

\subsection{Kinematics analysis for transmission angle}

When dealing with the transmission angle, it is suggested that the transmission angle of the fourbar linkage mechanism should be between $\left(45^{\circ}\right.$ $135^{\circ}$ ) so that the efficiency of the transmission angle of the four-bar linkage mechanism will be accepted [13]. In order to clarify this purpose, the transmission angle of the sets of four-bar linkages was calculated, as the shown in the figure (4).

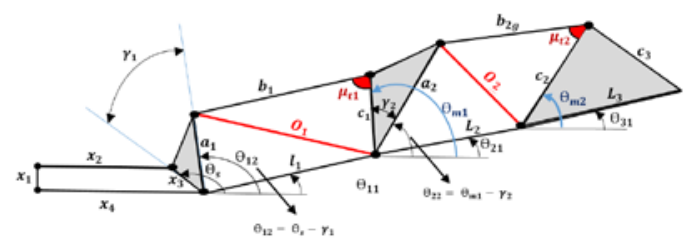

Figure (4): The transmission angles of the top \& bottom four bar linkages

The transmission angle of the first four-bar linkage can be written as:

$\mu_{t 1}=\cos ^{-1}\left[\frac{b_{1}^{2}+c_{1}^{2}-O_{1}^{2}}{2 b_{1} c_{1}}\right]$

Where:

$\left.\begin{array}{l}O_{1}^{2}=a_{1}^{2}+l_{1}^{2}-2 a_{1} l_{1} \cos \left(\theta_{12}-\theta_{11}\right) \\ \theta_{12}=\theta_{s}-\gamma_{1}\end{array}\right\}$

While the transmission angle of the second four-bar linkage mechanism can be expressed as:

$\mu_{t 2}=\cos ^{-1}\left[\frac{b_{2 g}^{2}+c_{2}^{2}-O_{2}^{2}}{2 b_{2 g} c_{2}}\right]$

Where:

$\left.\begin{array}{rl}O_{2}^{2} & =a_{2}^{2}+l_{2}^{2}-2 a_{2} l_{2} \cos \left(\theta_{22}-\theta_{21}\right) \\ \theta_{22} & =\theta_{m 1}-\gamma_{2}\end{array}\right\}$

In this study, The transmission angle in both mechanisms different from previous search (Ref [13]) equations because of the addition the angles of the ternary solid link $\left(\gamma_{1,2}\right)$.

\section{Multi objective function}

Optimization is maximizing some of functions or minimizing them relative to some of sets that represent the range of selections available in the problem situation. The gradient method consists of three steps of solution to find the optimal geometric parameters with high precision. At the first step, when the all possible solutions have been determined, the optimal design parameters will be calculated for each objective function. After finding the optimal solutions for each objective function, then the best solutions of all the objective functions will be chosen by collecting the minimum optimization formulation $G_{T}$, which is:

$$
\begin{gathered}
G_{T}=G_{1} \times w_{1}+G_{2} \times w_{2}+G_{3} \times w_{3}+G_{4} \times w_{4} \\
+G_{5} \times w_{5}
\end{gathered}
$$

Where:

$G_{T}$ : Optimization formulation.

$G_{n}$ : The objective function, $\quad n=1,2,3,4,5$

$w$ : The weight of the optimization.

The weight of the multi objectives optimization is assumed one for all objective functions $\left(w_{1}=w_{2}=w_{n}=1\right)$. When the first step is finished, the second step starts by searching for the optimum solution parameters in the optimal solution of the first step. Making new range depends on the optimal of the first step to start with the second step of optimization. The new range can be calculated using the following equations:

$$
M=(\operatorname{Max} . N o .-M i n . N o) \times \frac{1}{8}
$$

New range $=\left(\right.$ best of $1_{\text {st }}$ stage -

$M$, best of $1_{\text {st }}$ stage $\left.+M\right)$

The third step is the determination of whichever is the better of the two stages to achieve the optimal geometric parameters. Some of the parameters as the input parameters were been identified as listed table (1), which is illustrate the optimization input parameters. The parameter $l_{1}, l_{2} \& l_{3}$ was taken one and a half times of the original length of the human finger [15].

Table (1): Parameters of the optimization

\begin{tabular}{|c|c|c|c|}
\hline Par. & Unit & Min. & Max. \\
\hline $\boldsymbol{a}_{\mathbf{1}}$ & $\mathrm{mm}$ & 108 & 118 \\
\hline $\boldsymbol{b}_{\mathbf{1}}$ & $\mathrm{mm}$ & 85 & 95 \\
\hline $\boldsymbol{c}_{\mathbf{1}}$ & $\mathrm{mm}$ & 51 & 65 \\
\hline $\boldsymbol{a}_{\mathbf{2}}$ & $\mathrm{mm}$ & 65 & 78 \\
\hline $\boldsymbol{b}_{\mathbf{2}}$ & $\mathrm{mm}$ & 70 & 80 \\
\hline $\boldsymbol{c}_{\mathbf{2}}$ & $\mathrm{mm}$ & 28 & 37 \\
\hline $\boldsymbol{\gamma}_{\mathbf{1}}$ & Deg. & 30 & 60 \\
\hline $\boldsymbol{\gamma}_{\mathbf{2}}$ & Deg. & 30 & 60 \\
\hline $\boldsymbol{\theta}_{\mathbf{2}}$ & Deg. & 0 & 90 \\
\hline $\boldsymbol{\theta}_{\mathbf{3}}$ & Deg. & 0 & 90 \\
\hline $\boldsymbol{\theta}_{\mathbf{1 1}}$ & Deg. & 0 & 90 \\
\hline $\boldsymbol{x}_{\mathbf{2}}$ & $\mathrm{mm}$ & 70 & 80 \\
\hline $\boldsymbol{x}_{\mathbf{3}}$ & $\mathrm{mm}$ & 30 & 40 \\
\hline $\boldsymbol{l}_{\mathbf{1}}$ & $\mathrm{mm}$ & 64 & 64 \\
\hline $\boldsymbol{l}_{\mathbf{2}}$ & $\mathrm{mm}$ & 37 & 37 \\
\hline $\boldsymbol{l}_{\mathbf{3}}$ & $\mathrm{mm}$ & 34 & 34 \\
\hline $\boldsymbol{h}_{\mathbf{1}}$ & $\mathrm{mm}$ & 32 & 32 \\
\hline $\boldsymbol{h}_{\mathbf{2}}$ & $\mathrm{mm}$ & 18.5 & 18.5 \\
\hline $\boldsymbol{h}_{\mathbf{3}}$ & $\mathrm{mm}$ & 17 & 17 \\
\hline $\boldsymbol{\gamma}_{\mathbf{3}}$ & Deg. & 60 & 60 \\
\hline
\end{tabular}




\begin{tabular}{|c|c|c|c|}
\hline $\boldsymbol{x}_{\mathbf{1}}$ & $\mathrm{mm}$ & 40 & 40 \\
\hline $\boldsymbol{x}_{\mathbf{4}}$ & $\mathrm{mm}$ & 100 & 100 \\
\hline
\end{tabular}

\section{Geometric optimization}

It's important to have a good configuration of the robotic finger for its employment in grasping objects (to stratify forces to them), it's common to perform optimization process in function criteria's. Those criterions are derived from following equations: -

1. Grasping forces for Kino-static model (i.e. equation (6))

2. Kinematics analysis for grasping task (i.e. equations $(10 \& 19)$ )

3. kinematics analysis for transmission angle (i.e. equations ( $24 \& 27)$ )

It is very complicated to segregate each parameter because of the entanglement of the system. To fix the problem, a gradient descent method was chosen. Those criterias were defined to find the parameters:

\subsection{First criterion: The percentage of the grasping stability}

In order to characterize the capability for the under actuated finger in order to create the full grasp for the phalanx: [16]

$$
G_{1 n}=\frac{\int_{w} \delta\left(\theta^{*}, h^{*}\right) d h^{*} d \theta^{*}}{\int_{w} d h^{*} d \theta^{*}}
$$

Where $\delta\left(\theta^{*}, h^{*}\right)$ is a Kronecker - like symbol for positive of (f) vector that eliminates nonwhole-phalanx grasps:

$$
\delta\left(\theta^{*}, h^{*}\right)=\left\{\begin{array}{cc}
1 & \text { if } f_{i}>0 \\
0 & \text { otherwise }
\end{array}\right.
$$

And $\mathbf{W}$ is the workspace of the finger in terms of $\left(\theta^{*}, h^{*}\right)$, i.e the hyper parallelepiped defined by and $l_{i}>h_{i}>0 \& \frac{\pi}{2}>\theta_{i}>0, i>0$. This index physically represents the percentage of the stability that is achievable by full phalanx grasps, namely, whole-hand grasping workspace.

Minimizing the objective function will minimize this term $\left(G_{1}\right)$

$$
\left|G_{1}=100-G_{1 n}\right|
$$

\subsection{Second criterion: The grasp forces}

This is defined as the ratio of the total force on the three phalanges divided by the largest force as the equation below : [16]

$$
\begin{aligned}
G_{2 n}=\frac{f_{1}+f_{2}+f_{3}}{\max \left(f_{i}\right)} \quad \forall \theta_{i}, \quad i \\
=1,2,3
\end{aligned}
$$

In this study, to transform the objective function to minimum objective function there is need to deduct the above objective function from the maximum number, so:

$$
\left|G_{2}=3-G_{2 n}\right|
$$

\subsection{Third criterion: Squeezing force}

To assure the stability of the grasp, one needs a certain squeezing force. This force should be as high as possible, so [16]

$$
\begin{aligned}
& G_{3 n}=\frac{\left(f_{1}+f_{2}+f_{3}\right)_{\max }}{F_{a}} \quad \forall \theta_{i}, \quad i \\
& =1,2,3
\end{aligned}
$$

If the maximum squeezing force is $(14.82 \mathrm{~N})$ then the minimum objective function of the squeezing force is:[17]

$$
\left|G_{3}=14.82-G_{3 n}\right|
$$

\subsection{Fourth criterion: Mimic function for grasping task}

The objective function in this case, has to minimize the difference between the angles made by human and the robotic finger during grasping, which can be mathematically described as: [32]

$$
G_{4}=\left|\theta_{21 h}-\theta_{21}\right|+\left|\theta_{31 h}-\theta_{31}\right|
$$

The value of the angles that made by human finger: $\theta_{21 h}=325^{\circ}$ and $\theta_{31 h}=280^{\circ}$, which is for the cylindrical objects with radius $(3 \mathrm{~cm})$. [18]

\subsection{Fifth criterion: Transmission Angle for Grasping Task}

In generally, the best transmission angle for the four-bar linkages mechanism is $90^{\circ}$ therefore, to get the minimum objective function:[13]

$$
G_{5}=\left|\mu_{t 1 \min }-90^{\circ}\right|+\left|\mu_{t 2 \min }-90^{\circ}\right|
$$

\section{Principle of gradient descent method}

This method is one of the most exceedingly used approaches in the researches to solve the optimization problems [14]. The gradient descent method was chosen instead of other methods of optimization to find the optimal geometric parameters due to the large number of variables as listed in table (1) which requires the use of a computer with high efficiency and therefore relied on this method according to the possibilities available and also, this method gives the best solution with an acceptable period of time. It relies on the neighbored point by splitting the range that was selected to a number of segments. Also, it starts with creating all the possible solutions to find the objective function. Gradient descent method relies on checking for optimum geometric parameters in the neighbors of the 
initial range. The parameter will be splitted in to a number of neighbors, which is 4 segments. The process of gradient descent method starts with finding the optimum geometric parameters were chosen for each of five objective functions. Finally, chose the optimum geometric parameters from the best of five objective functions.

\section{Results and Discussion}

At the first stage of gradient descent method, the number of the overall possible solutions that created were $(1,594,323)$ solutions. First, the optimal geometric parameters will be found for each objective function. Then, the final optimal parameters for the first stage could be obtained from the best solutions that were calculated for each objective functions. Table (2) shows the values of optimum geometric parameters of each objective function and the final objective function. To start with the second stage of the optimization, new range should be taken to find all the possible solutions to get new optimal geometric parameters. The new range can be calculated by using the equation (29), therefore, the value of equation (29) is subtracted and collected from the best solution for the first stage by using the equation (30). The new range of the second stage is listed in the table (3). The number of the possible solutions that were calculated are $(1,594,323)$ solutions. Table (4) depicts the optimum geometric parameter for each objective function and the final optimum geometric parameter for the second stage. The solution optimization of the gradient descent method for the third stage was used to determine the final optimum geometric parameters from both the first and second stages, respectively. The number of possible solutions that generated are $(16,384)$ solutions. At third stage, the optimum geometric parameters are listed in table (5) showing are displayed that the final results are the same results of the second stage which indicate that the current results are the best for these conditions. The program's flow chart is shown in figure (5). The equation of Kino-static analysis was represented in equation (6) and calculated by using MATLAB program. Value of the input torque $t_{a}$ was assumed unity, and the value of the coefficient of friction was two. The grasping force of the robotic finger that obtained was influenced by two fundamental factors. Configurations of the finger were described by $\left(\theta_{2} \& \theta_{3}\right)$ and the location of the contact forces $\left(h_{1}, h_{2} \& h_{3}\right)$. The range of $\left(\theta_{2} \& \theta_{3}\right)$ is $0<\theta_{2}<90^{\circ}$ and $0<\theta_{3}<90^{\circ}$. Figure (6) explains the contact forces of the robotic finger. There are two regions, the first region is the stable region when all the three phalanges forces are positive. The second region is the unstable region when $f_{1} \& f_{2}$, are negative while $f_{3}$ is positive. Also, it can be seen that the increasing in $\theta_{2} \& \theta_{3}$ leads to increasing of $f_{1}$. The increasing in $\theta_{3}$ leads to increase of $f_{2}$, while the increasing of $\theta_{2}$ causes $f_{2}$ to be nearly constant with a little decrease near angle $90^{\circ} . f_{3}$ increases when there is increasing in $\theta_{2} \& \theta_{3}$. The grasping stability region when all the forces of the three phalanxes are positive is about $60 \%$ and the minimum optimization formulation $G_{T}$ is 19.75645 within 23 minutes by using personal computer.

Table (2): Optimum values of parameters of first stage

\begin{tabular}{|c|c|c|c|c|c|c|}
\hline \multirow{2}{*}{ Par. } & \multicolumn{7}{|c|}{ Objective Function } \\
\cline { 2 - 7 } & $G_{\mathbf{1}}$ & $G_{2}$ & $G_{\mathbf{3}}$ & $G_{\mathbf{4}}$ & $G_{\mathbf{5}}$ & Final \\
\hline $\boldsymbol{a}_{\mathbf{1}}$ & 109.6 & 113 & 116.3 & 116.3 & 109.6 & 116.3 \\
\hline $\boldsymbol{b}_{\mathbf{1}}$ & 86.6 & 86.6 & 86.6 & 90 & 86.6 & 90 \\
\hline $\boldsymbol{c}_{\mathbf{1}}$ & 53.3 & 62.6 & 62.6 & 58 & 53.3 & 62.6 \\
\hline $\boldsymbol{a}_{\mathbf{2}}$ & 67.16 & 67.16 & 75.8 & 75.83 & 67.1 & 75.8 \\
\hline $\boldsymbol{b}_{\mathbf{2}}$ & 71.6 & 71.6 & 71.6 & 78.3 & 71.6 & 78.3 \\
\hline $\boldsymbol{c}_{\mathbf{2}}$ & 29.5 & 29.5 & 35.5 & 35.5 & 29.5 & 35.5 \\
\hline $\boldsymbol{\gamma}_{\mathbf{1}}$ & 34.9 & 34.9 & 34.9 & 34.9 & 34.9 & 34.9 \\
\hline $\boldsymbol{\gamma}_{\mathbf{2}}$ & 34.9 & 34.9 & 34.9 & 34.9 & 34.9 & 34.9 \\
\hline $\boldsymbol{x}_{\mathbf{2}}$ & 71.6 & 71.6 & 71.6 & 71.6 & 71.6 & 71.6 \\
\hline $\boldsymbol{x}_{\mathbf{3}}$ & 31.6 & 31.6 & 31.6 & 38.3 & 31.6 & 38.3 \\
\hline $\boldsymbol{G}_{\boldsymbol{T}}$ & \multicolumn{7}{|c|}{} \\
\hline
\end{tabular}

Table (3): Geometrical parameters

\begin{tabular}{|c|c|c|c|}
\hline Par. & Unit & Min. & Max. \\
\hline $\boldsymbol{a}_{\mathbf{1}}$ & $\mathrm{mm}$ & 115.08 & 117.5 \\
\hline $\boldsymbol{b}_{\mathbf{1}}$ & $\mathrm{mm}$ & 88.7 & 91.2 \\
\hline $\boldsymbol{c}_{\mathbf{1}}$ & $\mathrm{mm}$ & 60.9 & 64.4 \\
\hline $\boldsymbol{a}_{\mathbf{2}}$ & $\mathrm{mm}$ & 74.2 & 77.4 \\
\hline $\boldsymbol{b}_{\mathbf{2}}$ & $\mathrm{mm}$ & 77.08 & 79.5 \\
\hline $\boldsymbol{c}_{\mathbf{2}}$ & $\mathrm{mm}$ & 34.3 & 36.6 \\
\hline $\boldsymbol{\gamma}_{\mathbf{1}}$ & Deg. & 31.2 & 38.7 \\
\hline $\boldsymbol{\gamma}_{\mathbf{2}}$ & Deg. & 31.2 & 38.7 \\
\hline $\boldsymbol{x}_{\mathbf{2}}$ & $\mathrm{mm}$ & 70.4 & 72.9 \\
\hline $\boldsymbol{x}_{\mathbf{3}}$ & $\mathrm{mm}$ & 37.08 & 39.5 \\
\hline
\end{tabular}

Table (4): Optimum values of geometrical parameters at second stage

\begin{tabular}{|c|c|c|c|c|c|c|}
\hline \multirow{2}{*}{ Par. } & \multicolumn{7}{|c|}{ Objective Function } \\
\cline { 2 - 7 } & $G_{\mathbf{1}}$ & $G_{2}$ & $G_{3}$ & $G_{\mathbf{4}}$ & $G_{5}$ & Final \\
\hline $\boldsymbol{a}_{\mathbf{1}}$ & 116.3 & 115.5 & 117.1 & 115.5 & 115.5 & 116.3 \\
\hline $\boldsymbol{b}_{\mathbf{1}}$ & 89.1 & 89.1 & 89.1 & 90.8 & 89.1 & 90.8 \\
\hline $\boldsymbol{c}_{\mathbf{1}}$ & 61.5 & 61.5 & 63.8 & 61.5 & 61.5 & 63.8 \\
\hline $\boldsymbol{a}_{\mathbf{2}}$ & 74.7 & 74.7 & 76.9 & 75.8 & 74.7 & 76.9 \\
\hline $\boldsymbol{b}_{\mathbf{2}}$ & 77.5 & 77.5 & 77.5 & 79.1 & 77.5 & 79.1 \\
\hline $\boldsymbol{c}_{\mathbf{2}}$ & 34.7 & 34.7 & 36.2 & 35.5 & 34.7 & 36.2 \\
\hline $\boldsymbol{\gamma}_{\mathbf{1}}$ & 32.4 & 32.4 & 32.4 & 32.4 & 32.4 & 32.4 \\
\hline $\boldsymbol{\gamma}_{\mathbf{2}}$ & 32.4 & 32.4 & 32.4 & 37.4 & 32.4 & 37.4 \\
\hline $\boldsymbol{x}_{\mathbf{2}}$ & 70.8 & 70.8 & 70.8 & 72.5 & 70.8 & 72.5 \\
\hline $\boldsymbol{x}_{\mathbf{3}}$ & 37.5 & 37.5 & 37.5 & 39.1 & 37.5 & 39.1 \\
\hline $\boldsymbol{G}_{\boldsymbol{T}}$ & \multicolumn{7}{|c|}{} \\
\hline
\end{tabular}


Table (5): Optimum values geometrical parameters at third stage

\begin{tabular}{|c|c|c|c|c|c|c|}
\hline \multirow{2}{*}{ Par. } & \multicolumn{7}{|c|}{ Objective Function } \\
\cline { 2 - 7 } & $G_{1}$ & $G_{2}$ & $G_{3}$ & $G_{\mathbf{4}}$ & $G_{5}$ & Final \\
\hline $\boldsymbol{a}_{\mathbf{1}}$ & 116.3 & 116.3 & 116.3 & 116.3 & 116.3 & 116.3 \\
\hline $\boldsymbol{b}_{\mathbf{1}}$ & 90 & 90 & 90 & 90.8 & 90 & 90.8 \\
\hline $\boldsymbol{c}_{\mathbf{1}}$ & 62.6 & 62.6 & 63.8 & 63.8 & 62.6 & 63.8 \\
\hline $\boldsymbol{a}_{\mathbf{2}}$ & 75.8 & 75.8 & 76.9 & 75.8 & 75.8 & 76.9 \\
\hline $\boldsymbol{b}_{\mathbf{2}}$ & 78.3 & 78.3 & 78.3 & 79.1 & 78.3 & 79.16 \\
\hline $\boldsymbol{c}_{\mathbf{2}}$ & 35.5 & 35.5 & 36.2 & 35.5 & 35.5 & 36.2 \\
\hline $\boldsymbol{\gamma}_{\mathbf{1}}$ & 34.9 & 34.9 & 34.9 & 32.4 & 34.9 & 32.4 \\
\hline $\boldsymbol{\gamma}_{\mathbf{2}}$ & 34.9 & 34.9 & 34.6 & 37.4 & 34.9 & 37.4 \\
\hline $\boldsymbol{x}_{\mathbf{2}}$ & 71.6 & 71.6 & 71.6 & 72.5 & 71.6 & 72.5 \\
\hline $\boldsymbol{x}_{\mathbf{3}}$ & 38.3 & 38.3 & 38.3 & 39.1 & 38.3 & 39.1 \\
\hline $\boldsymbol{G}_{\boldsymbol{T}}$ & \multicolumn{7}{|c|}{} \\
\hline
\end{tabular}

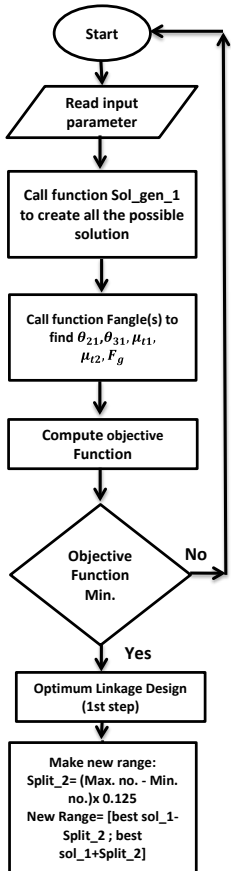

Figure (5): Flow chart of Program
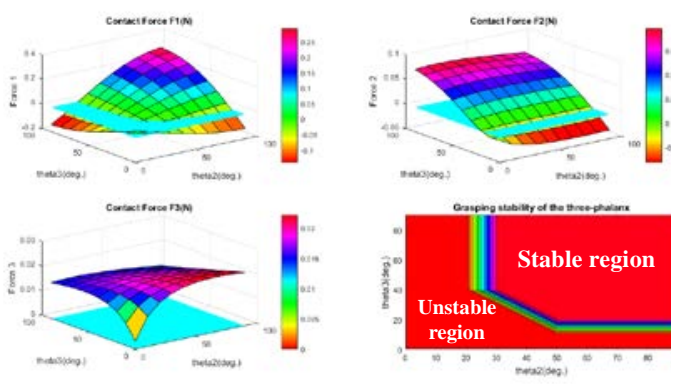

Figure (6): Contact forces and grasping stability of three phalanxes for the robotic finger with optimized parameters

\section{Manufacturing of Robotic Finger Prototype}

The sophisticated robotic finger in this work was built of hard plastic using rapid prototyping. This technique allowed the manufacture and demonstration of the prototypes within a short interval of time by using 3D Printers machine. 3D Printers give the ability to easily test product designs using models made of durable PLA (Polylactic acid) plastic. PLA plastic is tough sufficient to use it as working parts, as shown in figure (7). A series of examples of grasps that were performed with the underactuated robotic finger is shown in figure (8). The grasps are generally firm and stable. The finger was performed completely for enveloping grasps, which include the contact with all phalanges. The purpose of finger manufacturing was to achieve the results that appeared in the optimization program and to demonstrate its ability to grasp the objects without any hindrance. The robotic finger is capable of holding targets with different circular diameters. The robotic finger is capable of grasping objects with different circular diameters. Finally, the results have been shown that the robotic finger adapts to the wanted configurations.

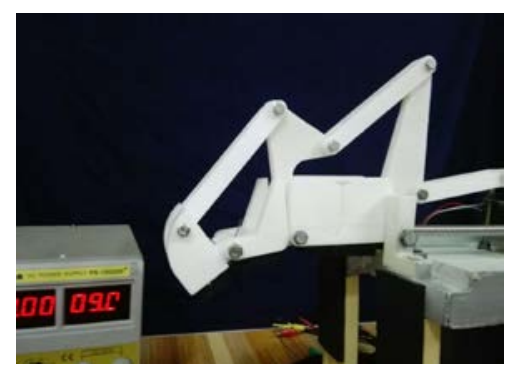

Figure (7) underactuated robotic finger
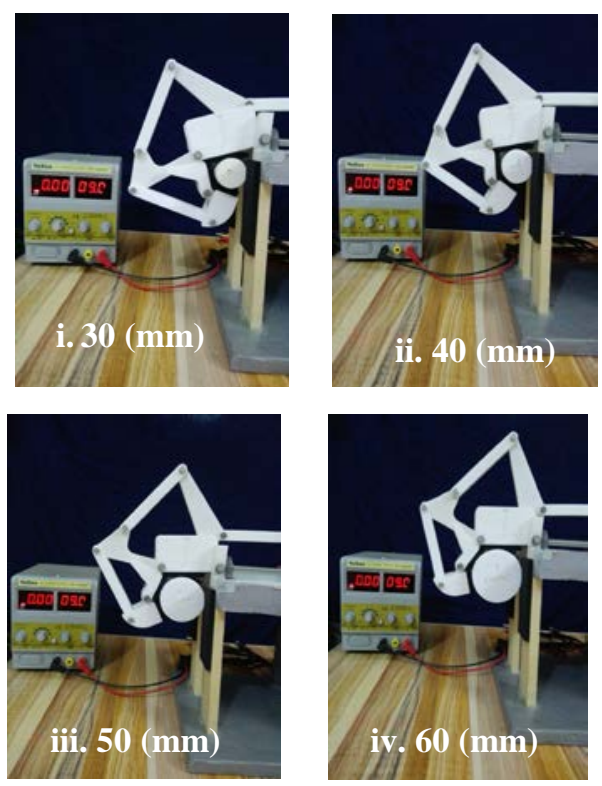

Figure (8): Grasping tasks of robotic finger for different objects 


\section{Conclusions}

1. New kinematic and kinetic equations for grasping finger were derived in this research taking into consideration the angle for the ternary solid links of the four-bar linkages.

2. Multi objectives function for robotic finger is optimized depending on five design criteria (percentage of the grasping stability, the grasp forces, squeezing force, Mimic function for grasping task, transmission angle for grasping task) to get an optimal solution, which leads to increase the finger performance at grasping tasks. The used optimization method consists of three steps to get a good accuracy of design parameters.

3. The results displayed that the gradient descent method is robust style to count the contact forces magnitude on the phalanxes in addition to the other objective functions.

4. Design of robotic finger with underactuated mechanism produces to minimize the cost and make the weight of the design more agreeable to use for industrial and prosthesis robotic hands that demand force for grasping operations.

5. The results exhibit that the adaptation of the finger is accepted, and the grasping forces are maximized and the stable area is $60 \%$ within the working region boundary.

Future work will develop the present robotic finger by increasing the tasks performed to do the pinching operation for different objects. This process is very important for the robotic finger to mimic the work of the human finger.

\section{Notations}

$$
\text { Symbol }
$$

$$
a_{1}, a_{2}, b_{1}, b_{2}
$$$$
, c_{1}, c_{2}
$$

Definition

The lengths of the corresponding bars.

\begin{tabular}{cc}
$\boldsymbol{f}$ & Grasping Force \\
$\boldsymbol{F}_{\boldsymbol{a}}$ & Actuator force \\
$\boldsymbol{f}_{\boldsymbol{t}}$ & Tangential force \\
$\boldsymbol{f}_{\boldsymbol{n}}$ & Normal force \\
$\boldsymbol{f}^{\boldsymbol{T}}$ & Transpose Force \\
$\boldsymbol{h}_{\mathbf{1}}, \boldsymbol{h}_{\mathbf{2}}, \boldsymbol{h}_{\mathbf{3}}$ & Grasping force location on \\
\multicolumn{3}{c}{ phalanx 1,2 and 3 respectively } \\
$\boldsymbol{J}_{\boldsymbol{w}}$ & Transmission matrix \\
$\boldsymbol{J}_{v}$ & Jacobian matrix of the finger \\
$\boldsymbol{l}_{\mathbf{1}}, \boldsymbol{l}_{\mathbf{2}}, \boldsymbol{l}_{\mathbf{3}}$ & Length of the proximal,
\end{tabular}

Middle and distal phalanx Input torque

Velocity vector of contact points

$\begin{array}{cr}x_{1}, x_{2}, & \text { The lengths of the } \\ x_{3}, x_{4} & \text { corresponding bars of slide }\end{array}$ mechanism

$\boldsymbol{\theta}_{\mathbf{1}}, \boldsymbol{\theta}_{\mathbf{2}} \quad$ The rotating angles of the , $\boldsymbol{\theta}_{3} \quad$ proximal phalanx, the middle phalanx and the distal phalanx,

\begin{tabular}{|c|c|}
\hline$\theta_{11}$ & $\begin{array}{l}\text { The angle of the } 1^{\text {st }} \text { phalanx } \\
l_{1}\end{array}$ \\
\hline$\theta_{12}$ & $\begin{array}{l}\text { The angle between } 1^{\text {st }} \\
\text { phalanx } l_{1} \text { and } a_{1}\end{array}$ \\
\hline$\theta_{21}$ & $\begin{array}{l}\text { The angle of the } 2^{\text {nd }} \text { phalanx } \\
l_{2}\end{array}$ \\
\hline $\boldsymbol{\theta}_{22}$ & $\begin{array}{l}\text { The angle between } 2^{\text {nd }} \\
\text { phalanx } l_{2} \text { and } a_{2}\end{array}$ \\
\hline$\theta_{13}$ & The angle of the link $b_{1}$ \\
\hline$\theta_{23}$ & The angle of the link $b_{2}$ \\
\hline$\theta_{31}$ & $\begin{array}{l}\text { The angle of the } 3^{\text {rd }} \text { phalanx } \\
l_{3}\end{array}$ \\
\hline$\theta_{21 h}, \theta_{31 h}$ & $\begin{array}{l}\text { Angles of human finger for } \\
\text { grasping task }\end{array}$ \\
\hline$\theta_{m 1}$ & $\begin{array}{l}\text { The output angle of the } 1^{\text {st }} \\
\text { four-bar linkages }\end{array}$ \\
\hline $\boldsymbol{\theta}_{m 2}$ & $\begin{array}{l}\text { The output angle of the } 2^{\text {nd }} \\
\text { four-bar linkages }\end{array}$ \\
\hline $\boldsymbol{\theta}_{\mathrm{s}}$ & $\begin{array}{l}\text { Output angle of slider } \\
\text { mechanism }\end{array}$ \\
\hline$\theta_{x 1}, \theta_{x 2}, \theta_{x 4}$ & $\begin{array}{l}\text { Angles of corresponding bars } \\
\text { of slider mechanism }\end{array}$ \\
\hline$\Psi_{1}, \Psi_{2}, \Psi_{3}$ & $\begin{array}{l}\text { The angle between the } \\
\text { driving bar and the proximal, } \\
\text { middle and distal phalanx }\end{array}$ \\
\hline $\begin{array}{c}\gamma_{1}, \gamma_{2}, \gamma_{3} \\
\mu\end{array}$ & $\begin{array}{l}\text { The angle of solid links } \\
\text { Coefficient of Friction }\end{array}$ \\
\hline$\mu_{t 1}, \mu_{t 2}$ & $\begin{array}{l}\text { The transmission angle for } \\
\text { the } 1^{\text {st }} \text { and } 2^{\text {nd }} \text { four-bar linkages } \\
\text { respectively }\end{array}$ \\
\hline
\end{tabular}
respectively

\section{References}

[1] W. Zhang, "Development of GestureChangeable under-actuated Humanoid Robotic Finger," Chinese J. Mech. Eng., vol. 23, no. 2, p. 142, 2010.

[2] C. M. M. Gosselin and T. Laliberté, "Underactuated mechanical finger with return actuation,” US Pat. 5,762,390, 1998.

[3] L. Birglen, "Underactuation in robotic grasping hands,” vol. 4, no. 3, pp. 1-11, 2002.

[4] N. E. N. Rodríguez, G. Carbone, and M. Ceccarelli, "Optimal design of driving mechanism in a 1-DOF anthropomorphic finger," Mech. Mach. Theory, vol. 41, no. 8, pp. 897-911, 2006.

[5] S. Yao, M. Ceccarelli, G. Carbone, and Z. Lu, "An optimal design for a new underactuated finger mechanism," in Proceedings of EUCOMES 2008 - The 2nd European 
Conference on Mechanism Science, 2009, pp. 149-157.

[6] W. ZHANG, D. ZHAO, H. ZHOU, Z. SUN, D. DU, and Q. CHEN, “Two-Dof Coupled and Self-Adaptive (Cosa) Finger: a Novel UnderActuated Mechanism,” Int. J. Humanoid Robot., vol. 10, no. 2, p. 1330001, 2013.

[7] J. Hsu, E. Yoshida, K. Harada, and A. Kheddar, "Self-locking Underactuated Mechanism for Robotic Gripper," 2016 IEEE Int. Conf. Adv. Intell. Mechatronics, pp. 620-627, 2017.

[8] J.-M. Boucher and L. Birglen, "Performance Augmentation of Underactuated Fingers' Grasps Using Multiple Drive Actuation,” Journal of Mechanisms and Robotics, vol. 9, no. 4. p. 41003, 2017.

[9] D. Zhao and W. Zhang, "Topology and analysis of three-phalanx COSA finger based on linkages for humanoid robot hands," Lect. Notes Comput. Sci. (including Subser. Lect. Notes Artif. Intell. Lect. Notes Bioinformatics), vol. 6424 LNAI, no. PART 1, pp. 465-476, 2010.

[10] L. Birglen and C. M. Gosselin, "Graspstate plane analysis of two-phalanx underactuated fingers," Mech. Mach. Theory, vol. 41, no. 7, pp. 807-822, 2006.

[11] S. S. Rattan, "Theory of Machines, Third Edit,” Tata McGraw Hill Educ. Priv. Ltd., 2009.
[12] N. Z. Azlan and Y. Hiroshi, "Underactuated anthropomorphic finger mechanism for grasping and pinching with optimized parameter," J. Comput. Sci., vol. 6, no. 8, pp. 928-933, 2010.

[13] Y. Liu, H. Wang, B. Li, and W. Zhou, "An optimal method to determine the parameters of anthropomorphic finger based on four-link mechanism," Proc. 2nd Bienn. IEEE/RAS-EMBS Int. Conf. Biomed. Robot. Biomechatronics, BioRob 2008, pp. 842-847, 2008.

[14] F. Andr, J. J. Quintero, and J. Carlos, "Convergence of the steepest descent method with line searches and uniformly convex objective in reflexive Banach,” vol. 20, pp. 161-173, 2015.

[15] P. Rea, "Mechatronic Object of A Pneumatic Drive Robotic Hand," PhD thesis. Univ. Cassino / Sch. Eng. Dep. Mech. Struct. Environ. Territ., 2013.

[16] L. Birglen, S. Member, and C. M. Gosselin, "Kinetostatic Analysis of Underactuated Fingers,” vol. 20, no. 2, pp. 211-221, 2004.

[17] L. P. Singh, "Work Study and Ergonomics,” Cambridge Univ., 2016.

[18] N. Zainul Azlan and H. Yamaura, "Anthropomorphic finger with optimized geometric parameters for pinching and grasping tasks," Mech. Mach. Theory, vol. 49, pp. 52-66, 2012.

\section{تحليل ألية إصبع رويوتي منخفض التشغيل لأمثل مسكة بأستخدام طريقة التدرج

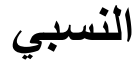

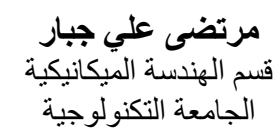

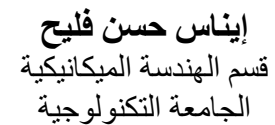

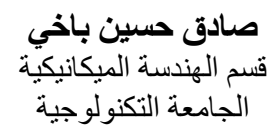

الخلاصة

تهتم هذه الدراسة بالتحقيق في المعلمات الهندسية المثلى للاصبع الروبوتي ذو ثلاث سلاميات منخفضة التشغيل. تم اشتقاق معادلات

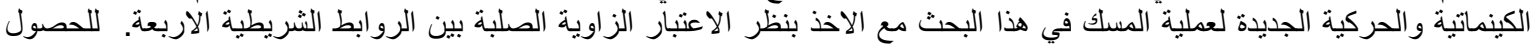

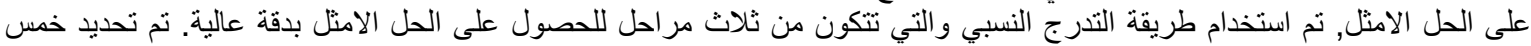

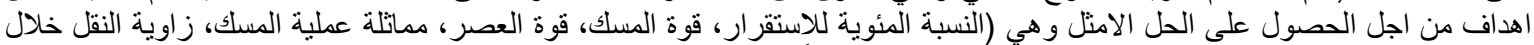

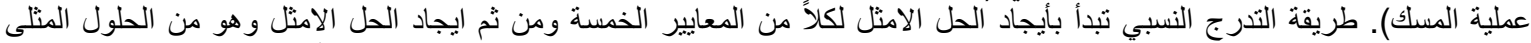

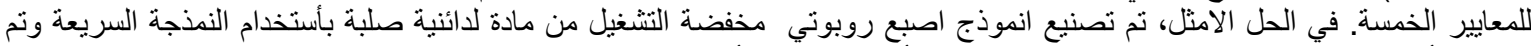

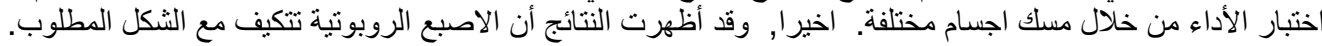

